\title{
A new full-NT step interior-point method for circular cone optimization
}

\author{
Behrouz Kheirfam* \\ Department of Applied Mathematics \\ Azarbaijan Shahid Madani University, Iran \\ E-mail: 〈b.kheirfam@azaruniv.ac.ir〉
}

\begin{abstract}
We present a full step feasible interior-point algorithm for circular cone optimization using Euclidean Jordan algebras. The specificity of our method is to use a transformation similar to that introduced by Darvay and Takács for the centering equations of the central path of the linear optimization. The Nesterov and Todd symmetrization scheme is used to derive the search directions. The theoretical complexity bound of the algorithm coincides with the best-known iteration bound for small-update methods.
\end{abstract}

Keywords: circular cone, Euclidean Jordan algebra, interior-point methods

Received: April 14, 2018; accepted: October 22, 2019; available online: December 13, 2019

DOI: $10.17535 /$ crorr.2019.0023

\section{Introduction}

Interior-point methods (IPMs) that initiated by the landmark paper of Karmarkar [9] play a fundamental role in moder optimization. They attracted the attention of many researchers due to the polynomial complexity and their practical effect. For instance, Illés and Terlaky [8] presented a comparison between the IPMs and pivot methods from the practical and theoretical point of view. Monteiro [13] introduced primal-dual path-following algorithms for solving semidefinite optimization (SDO). Alizadeh and Goldfarb [2] proposed primal-dual path-following algorithms for second-order cone optimization (SOCO). The primal-dual pathfollowing algorithms have been extended by Schmieta and Alizadeh [15] to symmetric cone optimization (SCO) using Euclidean Jordan algebras.

The full-Newton step primal-dual path-following IPM was first introduced by Roos et al. [14]. Later on, De Klerk [6], Wang et al. [20], Wang and Lesaja [16] and Kheirfam [11] extended the results for LO to SDO and $P_{*}(\kappa)$-linear complementarity problem, shortly $P_{*}(\kappa)$-LCP, the Cartesian $P_{*}(\kappa)$-LCP over symmetric cone $P_{*}(\kappa)$-SCLCP and circular cone optimization CCO. Based on a new proximity measure, Zhang et al. [22] and Kheirfam [10] respectively generalized Roos et al.'s algorithm to SDO and $P_{*}(\kappa)$-HLCP. In the 2003s, Darvay [4] introduces a modification in the centering equations $x s=\mu e$ of the central path by considering $\varphi\left(\frac{x s}{\mu}\right)=$ $\varphi(e)$, where $\varphi: R_{+}^{n} \rightarrow R_{+}^{n}$ is assumed to be a smooth function such that $\varphi(0)=0$. This direction has become an active area in the past few years for the case $\varphi(t)=\sqrt{t}$. For example, the Darvay's results for LO is extended to convex quadratic optimization (CQO) [1], SDO [18], $P_{*}(\kappa)$-LCP [21], SOCO [17] and SCO [19]. Very recently, Darvay and Takács [5] introduce another method for characterizing search directions for LO. They replace the centering equations

${ }^{*}$ Corresponding author. 
$x s=\mu e$ by $\varphi\left(\frac{x s}{\mu}\right)=\varphi\left(\sqrt{\frac{x s}{\mu}}\right)$, and then apply Newton's method in order to get the new search directions for the case $\varphi(t)=t^{2}$.

As mentioned above, the extension of the algorithms proposed for LO to more general optimization problems such as LCP, $P_{*}(\kappa)-\mathrm{LCP}, \mathrm{SDO}, \mathrm{SOCO}, \mathrm{SCO}$ is a very active area of research during the past years. The applications of the circular cone appear in many realworld engineering problems such as grasp optimizationand the optimal grasping manipulation problems for multi-fingered robots [12]. However, there is little work on the algorithms for solving the CCO. These motivate us to generalize Darvay and Takács's technique [5] for LO to $\mathrm{CCO}$. We prove that the proposed algorithm is well-defined and derive worst-case complexity bound for our algorithm.

The paper is organized as follows. In Section 2, we present the state of the art of CCO and related Euclidean Jordan algebra. In Section 3, we study the central path and the new search directions for CCO. Then we present a feasible full-NT step algorithm for CCO. In Section 4, we show the polynomial complexity of our algorithm. Finally, some conclusions are given in Section 5 .

The following notations are used throughout this paper. Let $R^{n}$ denotes the space of $n$ dimensional real column vectors. We use $x=\left(x_{0} ; \bar{x}\right)$ for the column vector $x=\left(x_{0}, \bar{x}^{T}\right)^{T} \in$ $R \times R^{n-1}$, and use $(x ; y ; s)$ for adjoining vectors $x, y, s$ in a column $\left(x^{T}, y^{T}, s^{T}\right)^{T}$. The symbols $\|\cdot\|$ and $\|\cdot\|_{\theta, F}$ respectively denote the standard Euclidean norm and the Frobenius norm. If $A \subseteq R^{k}$ and $B \subseteq R^{l}$, then

$$
A \times B=\{(x ; y): x \in A, y \in B\}
$$

is their Cartesian product. The $\operatorname{int} A$ denotes the interior of $A$.

\section{CCO and related Jordan algebra}

Consider the $\mathrm{CCO}$ in the standard form

$$
\min \left\{\sum_{j=1}^{N}\left\langle c^{j}, x^{j}\right\rangle_{\theta_{j}}: \sum_{j=1}^{N}\left(A_{j} x^{j}\right)_{\theta_{j}}=b, x^{j} \in \mathcal{Q}_{\theta_{j}}^{n_{j}}\right\},
$$

where $b \in R^{m}, A_{j} \in R^{m \times n_{j}}, c^{j} \in R^{n_{j}}$, and $\mathcal{Q}_{\theta_{j}}^{n_{j}}$ is the circular cone of dimension $n_{j}$ defined by

$$
\mathcal{Q}_{\theta_{j}}^{n_{j}}:=\left\{\left(x_{0}^{j} ; \bar{x}^{j}\right): x_{0}^{j} \geq \cot \left(\theta_{j}\right)\left\|\bar{x}^{j}\right\|\right\}
$$

for $\theta_{j} \in\left(0, \frac{\pi}{2}\right), j=1, \ldots, N$, and $\|\cdot\|$ refers to the Euclidean norm. Moreover, $\left\langle c^{j}, x^{j}\right\rangle_{\theta_{j}}:=$ $\left(c^{j}\right)^{T} I_{\theta_{j}, n_{j}}^{2} x^{j},\left(A_{j} x^{j}\right)_{\theta_{j}}:=A_{j} I_{\theta_{j}, n_{j}}^{2} x^{j}$ with

$$
I_{\theta_{j}, n_{j}}:=\left[\begin{array}{lc}
1 & 0^{T} \\
0 & \cot \left(\theta_{j}\right) I_{n_{j}-1}
\end{array}\right] \in R^{n_{j} \times n_{j}}, \quad j=1, \ldots, N
$$

which respectively denote the circular inner product between $c^{j}$ and $x^{j}$ and the circular matrixvector product between $A_{j}$ and $x^{j}$ with the circular identity matrix $I_{\theta_{j}, n_{j}}$. Let us consider

$$
\begin{gathered}
A=\left[A_{1}, A_{2}, \ldots, A_{N}\right] \in R^{m \times n}, c=\left(c^{1} ; c^{2} ; \ldots ; c^{N}\right) \in R^{n_{1}} \times \cdots \times R^{n_{N}}=R^{n}, \\
I_{\theta, n}=\left[\begin{array}{ccc}
I_{\theta_{1}, n_{1}} & & \\
& \ddots & \\
& & I_{\theta_{N}, n_{N}}
\end{array}\right]_{n \times n} \text { and } x=\left(x^{1} ; x^{2} ; \ldots ; x^{N}\right) \in \mathcal{Q}_{\theta_{1}}^{n_{1}} \times \cdots \times \mathcal{Q}_{\theta_{N}}^{n_{N}}:=\mathcal{Q}_{\theta}^{n},
\end{gathered}
$$


where $n=\sum_{j=1}^{N} n_{j}$. Then, the problem $(\mathrm{P})$ can be simply written as

$$
\min \left\{\langle c, x\rangle_{\theta}:(A x)_{\theta}=b, x \in \mathcal{Q}_{\theta}^{n}\right\} \text {. }
$$

The dual of $(\mathrm{P})$ is defined by

$$
\max \left\{b^{T} y: A^{T} y+s=c, y \in R^{m}, s \in \mathcal{Q}_{\theta}^{n}\right\} .
$$

We assume that $A$ has full row rank; i.e., $\operatorname{rank}(A)=m$, and both primal and dual problems satisfy the interior-point condition (IPC); i.e., there exists a primal-feasible vector $x$ with $x \in$ $\operatorname{int} \mathcal{Q}_{\theta}^{n}$ and a dual-feasible $y$ and $s$ such that $s \in \operatorname{int} \mathcal{Q}_{\theta}^{n}$. It is also worth noting that CCO includes second-order cone optimization as a special case. Here, for the sake of simplicity, we assume that $N=1$, hence $\mathcal{Q}_{\theta_{1}}^{n_{1}}=\mathcal{Q}_{\theta}^{n}$. Let $\mathcal{E}^{n}$ denote the $n$ dimensional real vector space $R \times R^{n-1}$ whose elements $x$ are indexed with 0 . Note that for two vectors $x, y \in \mathcal{E}^{n}$, we can rewrite their circular inner product as follows

$$
\langle x, y\rangle_{\theta}=x^{T} I_{\theta, n}^{2} y=x_{0} y_{0}+\cot ^{2}(\theta) \bar{x}^{T} \bar{y}
$$

Let $x \in \mathcal{E}^{n}$. The circular spectral decomposition of $x$ with respect to the angle $\theta \in\left(0, \frac{\pi}{2}\right)$ is defined as follows [3]

$$
x=\lambda_{\theta, \max }(x) c_{\theta, 1}(x)+\lambda_{\theta, \min }(x) c_{\theta, 2}(x),
$$

where $\lambda_{\theta, \max }=x_{0}+\cot (\theta)\|\bar{x}\|, \lambda_{\theta, \min }=x_{0}-\cot (\theta)\|\bar{x}\|$ are the eigenvalues of $x$ and their associated eigenvectors given by

$$
c_{\theta, 1}(x)=\frac{1}{2}\left(1 ; \frac{\tan (\theta) \bar{x}}{\|\bar{x}\|}\right), c_{\theta, 2}(x)=\frac{1}{2}\left(1 ; \frac{-\tan (\theta) \bar{x}}{\|\bar{x}\|}\right) .
$$

Under Spectral Decomposition, we have

$$
\operatorname{tr}(x)=\lambda_{\theta, \min }(x)+\lambda_{\theta, \max }(x)=2 x_{0}, \operatorname{det}_{\theta}(x)=\lambda_{\theta, \min }(x) \lambda_{\theta, \max }(x)=x_{0}^{2}-\cot ^{2}(\theta)\|\bar{x}\|^{2} .
$$

As a result it can be easily seen that $c_{\theta, 1}(x)+c_{\theta, 2}(x)=e:=(1 ; 0)$, which is the identity element of $\mathcal{E}^{n}, \operatorname{trace}(e)=2$ and $\operatorname{det}(x)=1$. For any real-valued continuous function $f$, we define the image of $x$ under $f$ with respect to $\theta$ as

$$
f(x):=f\left(\lambda_{\theta, \max }(x)\right) c_{\theta, 1}(x)+f\left(\lambda_{\theta, \min }(x)\right) c_{\theta, 2}(x),
$$

in particular, we get $x^{-1}=\lambda_{\theta, \max }^{-1}(x) c_{\theta, 1}(x)+\lambda_{\theta, \min }^{-1}(x) c_{\theta, 2}(x)$. The Frobenius norm with respect to $\theta$ of $x$ is defined as $\|x\|_{\theta, F}:=\sqrt{\lambda_{\theta, \min }^{2}(x)+\lambda_{\theta, \max }^{2}(x)}$. The arrow-shaped matrix $\operatorname{Arw}_{\theta}(x)$ associated with $x$ in $\mathcal{E}^{n}$ is defined as [3]

$$
\operatorname{Arw}_{\theta}(x):=\left[\begin{array}{cc}
x_{0} & \cot ^{2}(\theta) \bar{x}^{T} \\
\bar{x} & x_{0} I_{n-1}
\end{array}\right]
$$

Observe that $x \in \mathcal{Q}_{\theta}^{n},\left(x \in \operatorname{int} \mathcal{Q}_{\theta}^{n}\right)$ if and only if $\operatorname{Arw}_{\theta}(x)$ is positive semidefinite (positive definite); i.e., $\operatorname{Arw}_{\theta}(x) \succcurlyeq 0\left(\operatorname{Arw}_{\theta}(x) \succ 0\right)$. For any $x, s \in \mathcal{E}^{n}$, the Jordan multiplication with respect to $\theta$ is defined as

$$
(x \circ s)_{\theta}=\left(\langle x, s\rangle_{\theta} ; x_{0} \bar{s}+s_{0} \bar{x}\right)=\operatorname{Arw}_{\theta}(x) s=\operatorname{Arw}_{\theta}(x) \operatorname{Arw}_{\theta}(s) e
$$

One can easily verify that $\left(\mathcal{E}^{n}, \theta, \circ\right)$ is a Euclidean Jordan algebra under the circular inner product $\langle\cdot, \cdot\rangle_{\theta}$ with $e=(1 ; 0) \in \mathcal{E}^{n}$; i.e., for $x, s, y \in \mathcal{E}^{n},(x \circ s)_{\theta}=(s \circ x)_{\theta},\left(x \circ\left(x^{2} \circ s\right)_{\theta}\right)_{\theta}=$ $\left(x^{2} \circ(x \circ s)_{\theta}\right)_{\theta}$ where $x^{2}=(x \circ x)_{\theta}$, and $\left\langle(x \circ s)_{\theta}, y\right\rangle_{\theta}=\left\langle x,(s \circ y)_{\theta}\right\rangle_{\theta}$ and $(x \circ e)_{\theta}=(e \circ x)_{\theta}=x$. 
Moreover, we have $c_{\theta, 1}^{2}=\left(c_{\theta, 1} \circ c_{\theta, 1}\right)_{\theta}=c_{\theta, 1}$ and $c_{\theta, 2}^{2}=\left(c_{\theta, 2} \circ c_{\theta, 2}\right)_{\theta}=c_{\theta, 2}$ and $\left(c_{\theta, 1} \circ c_{\theta, 2}\right)_{\theta}=0$. Therefore, $\left\{c_{\theta, 1}, c_{\theta, 2}\right\}$ is a Jordan frame. We say that two vectors $x$ and $s$ in $\mathcal{E}^{n}$ are similar, denoted by $x \sim s$, if they share a Jordan frame. The cone of squares of the Euclidean Jordan algebra $\left(\mathcal{E}^{n}, \theta, \circ\right)$ is the circular cone $\mathcal{Q}_{\theta}^{n}\left[3\right.$, Theorem 4]. The quadratic representation $P_{\theta}(x)$ associated with $x \in \mathcal{E}^{n}$ with respect to $\theta$ is given as $P_{\theta}(x)=2 \operatorname{Arw}_{\theta}^{2}(x)-\operatorname{Arw}_{\theta}\left(x^{2}\right)$. In particular, one has $P_{\theta}(x) e=x^{2}, P_{\theta}(x) x^{-1}=x$ and $P_{\theta}\left(x^{-1}\right) P_{\theta}(x)=P_{\theta}(x) P_{\theta}\left(x^{-1}\right)=I_{n}$, i.e., $P_{\theta}\left(x^{-1}\right)=P_{\theta}(x)^{-1}[11]$. In the sequel, we generalize the above notions and concepts to the case where $N>1$, when $\mathcal{E}^{n}=\mathcal{E}^{n_{1}} \times \cdots \times \mathcal{E}^{n_{N}}$ and the circular cone underlying $\mathcal{Q}_{\theta}^{n}$ is the Cartesian product on $N$ circular cones; i.e., $\mathcal{Q}_{\theta}^{n}=\mathcal{Q}_{\theta_{1}}^{n_{1}} \times \cdots \times \mathcal{Q}_{\theta_{N}}^{n_{N}}$. The Jordan algebra associated with the circular cone $\mathcal{Q}_{\theta}^{n}$ is given by

$$
(x \circ s)_{\theta}=\left(\left(x^{1} \circ s^{1}\right)_{\theta_{1}} ; \ldots ;\left(x^{N} \circ s^{N}\right)_{\theta_{N}}\right),
$$

with $e=\left(e^{1} ; \ldots ; e^{N}\right)$ being its identity element. Note that $\operatorname{tr}(e)=2 N$. The arrow-shaped matrix $\operatorname{Arw}_{\theta}(x)$ and the quadratic representation $P_{\theta}(x)$ of the Jordan algebra $\left(\mathcal{E}^{n}, \theta, \circ\right)$ with respect to $\theta$ can be respectively adjusted to

$$
\operatorname{Arw}_{\theta}(x):=\operatorname{diag}\left(\operatorname{Arw}_{\theta_{1}}\left(x^{1}\right), \ldots, \operatorname{Arw}_{\theta_{N}}\left(x^{N}\right)\right), P_{\theta}(x):=\operatorname{diag}\left(P_{\theta_{1}}\left(x^{1}\right), \ldots, P_{\theta_{N}}\left(x^{N}\right)\right) .
$$

Furthermore

$$
\lambda_{\theta, \max }(x)=\max _{1 \leq j \leq N} \lambda_{\theta_{j}, \max }\left(x^{j}\right), \lambda_{\theta, \min }(x)=\min _{1 \leq j \leq N} \lambda_{\theta_{j}, \min }\left(x^{j}\right),
$$

and

$$
\|x\|_{\theta, F}^{2}=\sum_{j=1}^{N}\left\|x^{j}\right\|_{\theta_{j}, F}^{2}, \quad \operatorname{tr}(x)=\sum_{j=1}^{N} \operatorname{tr}\left(x^{j}\right) .
$$

\section{Feasible full-NT step IPM}

In this section, we first introduce the concept of the central path for $\mathrm{CCO}$, and then generalize Darvay and Takács's technique [5] for LO to CCO.

\subsection{Central path}

The Karush-Kuhn-Tucker (KKT) optimality conditions for problems (P) and (D) is stated as follows:

$$
\begin{aligned}
(A x)_{\theta} & =b, \quad x \in \mathcal{Q}_{\theta}^{n}, \\
A^{T} y+s & =c, \quad s \in \mathcal{Q}_{\theta}^{n}, \\
(x \circ s)_{\theta} & =0 .
\end{aligned}
$$

The first two equations represent primal and dual feasibility and the third equation is called the complementarity condition for (P) and (D). According to the key idea of IPMs, we replace the complementarity condition $(x \circ s)_{\theta}=0$ by the parameterized equation $(x \circ s)_{\theta}=\mu_{\theta} e$ with $\mu_{\theta}>0$. This leads to the following system

$$
\begin{aligned}
(A x)_{\theta} & =b, \quad x \in \operatorname{int} \mathcal{Q}_{\theta}^{n}, \\
A^{T} y+s & =c, \quad s \in \operatorname{int} \mathcal{Q}_{\theta}^{n}, \\
(x \circ s)_{\theta} & =\mu_{\theta} e .
\end{aligned}
$$

Since the IPC holds and $A$ has full rank, the system (4) has a unique solution, denoted by $\left(x\left(\mu_{\theta}\right), y\left(\mu_{\theta}\right), s\left(\mu_{\theta}\right)\right)$ for each $\mu_{\theta}>0$. We call $x\left(\mu_{\theta}\right)$ the $\mu_{\theta}$-center of $(\mathrm{P})$ and $\left(y\left(\mu_{\theta}\right), s\left(\mu_{\theta}\right)\right)$ the 
$\mu_{\theta}$-center of (D). The set of all $\mu_{\theta}$-centers is called the central path. It is worth noting that at the $\mu_{\theta}$-center, due to the definition of the trace function, together with (2), we get

$$
\left\langle x\left(\mu_{\theta}\right), s\left(\mu_{\theta}\right)\right\rangle_{\theta}=\frac{1}{2} \operatorname{tr}\left(\left(x\left(\mu_{\theta}\right) \circ s\left(\mu_{\theta}\right)\right)_{\theta}\right)=\frac{1}{2} \mu_{\theta} \operatorname{tr}(e)=N \mu_{\theta} .
$$

It is clear to see that if $\mu_{\theta}$ goes to zero, then the limit points satisfy the complementarity condition $(x \circ s)_{\theta}=0$, and yields an optimal solution for $(\mathrm{P})$ and $(\mathrm{D})$. Let us consider $u \in \operatorname{int} \mathcal{Q}_{\theta}^{n}$. It is a well known fact that $(x \circ s)_{\theta}=\mu_{\theta} e$ if and only if $\left(P_{\theta}(u) x \circ P_{\theta}\left(u^{-1}\right) s\right)_{\theta}=\mu_{\theta} e[15$, cf.Lemma 28]. In this way, we see that system (4) can be rewritten in the following equivalent form:

$$
\begin{aligned}
(A x)_{\theta} & =b, \quad x \in \operatorname{int} \mathcal{Q}_{\theta}^{n}, \\
A^{T} y+s & =c, \quad s \in \operatorname{int} \mathcal{Q}_{\theta}^{n}, \\
\left(P_{\theta}(u) x \circ P_{\theta}\left(u^{-1}\right) s\right)_{\theta} & =\mu_{\theta} e
\end{aligned}
$$

For each choice of $u$ and applying Newton's method to system (5) one gets a different direction. In this article, we will pay particular attention to the case of Nesterov-Todd (NT) direction; i.e., $u=w^{-\frac{1}{2}}$ with

$$
w=P_{\theta}\left(x^{\frac{1}{2}}\right)\left(P_{\theta}\left(x^{\frac{1}{2}}\right) s\right)^{-\frac{1}{2}}\left[=P_{\theta}\left(s^{-\frac{1}{2}}\right)\left(P_{\theta}\left(s^{\frac{1}{2}}\right) x\right)^{\frac{1}{2}}\right]
$$

which is called the scaling point of $x$ and $s$. Let us define

$$
v_{\theta}=\frac{P_{\theta}\left(w^{-\frac{1}{2}}\right) x}{\sqrt{\mu_{\theta}}}\left[=\frac{P_{\theta}\left(w^{\frac{1}{2}}\right) s}{\sqrt{\mu_{\theta}}}\right] .
$$

Then, by using the third equation of (5), we have

$$
v_{\theta}^{2}=e \Leftrightarrow v_{\theta}=e \Leftrightarrow v_{\theta}^{2}=v_{\theta} .
$$

\subsection{Search directions}

Here, we present a class of search directions for CCO based on Darvay and Takács's technique for LO [5]. Consider the univariate function $\varphi$ continuously differentiable on $\left(\sigma^{2}, \infty\right)$ with $0 \leq \sigma<1$, such that $2 t \varphi^{\prime}\left(t^{2}\right)-\varphi^{\prime}(t)>0$ for each $t>\sigma^{2}$. Due to (6), we replace the standard centering equation $\left(P_{\theta}\left(w^{-\frac{1}{2}}\right) x \circ P_{\theta}\left(w^{\frac{1}{2}}\right) s\right)_{\theta}=\mu_{\theta}$ e by

$$
\varphi\left(\left(\left(\frac{P_{\theta}\left(w^{-\frac{1}{2}}\right) x}{\sqrt{\mu_{\theta}}}\right) \circ\left(\frac{P_{\theta}\left(w^{\frac{1}{2}}\right) s}{\sqrt{\mu_{\theta}}}\right)\right)_{\theta}\right)=\varphi\left(\left(\left(\frac{P_{\theta}\left(w^{-\frac{1}{2}}\right) x}{\sqrt{\mu_{\theta}}}\right) \circ\left(\frac{P_{\theta}\left(w^{\frac{1}{2}}\right) s}{\sqrt{\mu_{\theta}}}\right)\right)_{\theta}^{\frac{1}{2}}\right) .
$$

Therefore, the system (5) can be rewritten in the following scaled form:

$$
\begin{gathered}
A I_{\theta, n}^{2} P_{\theta}\left(w^{\frac{1}{2}}\right) P_{\theta}\left(w^{-\frac{1}{2}}\right) x=b \\
I_{\theta, n}^{2} P_{\theta}\left(w^{\frac{1}{2}}\right) A^{T} y+I_{\theta, n}^{2} P_{\theta}\left(w^{\frac{1}{2}}\right) s=I_{\theta, n}^{2} P_{\theta}\left(w^{\frac{1}{2}}\right) c \\
\varphi\left(\left(\left(\frac{P_{\theta}\left(w^{-\frac{1}{2}}\right) x}{\sqrt{\mu_{\theta}}}\right) \circ\left(\frac{P_{\theta}\left(w^{\frac{1}{2}}\right) s}{\sqrt{\mu_{\theta}}}\right)\right)_{\theta}\right)=\varphi\left(\left(\left(\frac{P_{\theta}\left(w^{-\frac{1}{2}}\right) x}{\sqrt{\mu_{\theta}}}\right) \circ\left(\frac{P_{\theta}\left(w^{\frac{1}{2}}\right) s}{\sqrt{\mu_{\theta}}}\right)\right)_{\theta}^{\frac{1}{2}}\right),
\end{gathered}
$$

or simplicity,

$$
\begin{aligned}
\tilde{A} \tilde{x} & =b \\
\tilde{A}^{T} y+I_{\theta, n}^{2} \tilde{s} & =\tilde{c} \\
\varphi\left(\left(\left(\frac{\tilde{x}}{\sqrt{\mu_{\theta}}}\right) \circ\left(\frac{\tilde{s}}{\sqrt{\mu_{\theta}}}\right)\right)_{\theta}\right) & =\varphi\left(\left(\left(\frac{\tilde{x}}{\sqrt{\mu_{\theta}}}\right) \circ\left(\frac{\tilde{s}}{\sqrt{\mu_{\theta}}}\right)\right)_{\theta}^{\frac{1}{2}}\right),
\end{aligned}
$$


where $\tilde{A}=A I_{\theta, n}^{2} P_{\theta}\left(w^{\frac{1}{2}}\right), \tilde{x}=P_{\theta}\left(w^{-\frac{1}{2}}\right) x, \tilde{s}=P_{\theta}\left(w^{\frac{1}{2}}\right) s, \tilde{c}=I_{\theta, n}^{2} P_{\theta}\left(w^{\frac{1}{2}}\right) c$. If we define

$$
f(\tilde{x}, y, \tilde{s}):=\left[\begin{array}{c}
\tilde{A} \tilde{x}-b \\
\tilde{A}^{T} y+I_{\theta, n}^{2} \tilde{s}-\tilde{c} \\
\varphi\left(\left(\left(\frac{\tilde{x}}{\sqrt{\mu_{\theta}}}\right) \circ\left(\frac{\tilde{s}}{\sqrt{\mu_{\theta}}}\right)\right)_{\theta}\right)-\varphi\left(\left(\left(\frac{\tilde{x}}{\sqrt{\mu_{\theta}}}\right) \circ\left(\frac{\tilde{s}}{\sqrt{\mu_{\theta}}}\right)\right)_{\theta}^{\frac{1}{2}}\right)
\end{array}\right],
$$

then system (8) can be written as follows: $f(\tilde{x}, y, \tilde{s})=0$. A Newton method applied to this system leads to the following linear system:

$$
\begin{gathered}
\tilde{A} \Delta \tilde{x}=b-\tilde{A} \tilde{x} \\
\tilde{A}^{T} \Delta y+I_{\theta, n}^{2} \Delta \tilde{s}=\tilde{c}-\tilde{A}^{T} y-I_{\theta, n}^{2} \tilde{s} \\
\varphi\left(\left(\left(\frac{\tilde{x}+\Delta \tilde{x}}{\sqrt{\mu_{\theta}}}\right) \circ\left(\frac{\tilde{s}+\Delta \tilde{s}}{\sqrt{\mu_{\theta}}}\right)\right)_{\theta}\right)-\varphi\left(\left(\left(\frac{\tilde{x}+\Delta \tilde{x}}{\sqrt{\mu_{\theta}}}\right) \circ\left(\frac{\tilde{s}+\Delta \tilde{s}}{\sqrt{\mu_{\theta}}}\right)\right)_{\theta}^{\frac{1}{2}}\right)=0,
\end{gathered}
$$

where $\Delta \tilde{x}=P_{\theta}\left(w^{-\frac{1}{2}}\right) \Delta x$ and $\Delta \tilde{s}=P_{\theta}\left(w^{\frac{1}{2}}\right) \Delta s$. From Corollary 2.14 in [17] and the fact that $\tilde{x}$ and $(y, \tilde{s})$ are feasible, we get

$$
\begin{gathered}
\tilde{A} \Delta \tilde{x}=0 \\
\tilde{A}^{T} \Delta y+I_{\theta, n}^{2} \Delta \tilde{s}=0 \\
\frac{1}{\mu_{\theta}}\left((\tilde{x} \circ \Delta \tilde{s})_{\theta}+(\Delta \tilde{x} \circ \tilde{s})_{\theta}\right)=(\tilde{z} \circ \underline{z})_{\theta},
\end{gathered}
$$

where

$$
\tilde{z}=\left[\varphi^{\prime}\left(\left(\left(\frac{\tilde{x}}{\sqrt{\mu_{\theta}}}\right) \circ\left(\frac{\tilde{s}}{\sqrt{\mu_{\theta}}}\right)\right)_{\theta}\right)-\frac{1}{2}\left(\left(\left(\frac{\tilde{x}}{\sqrt{\mu_{\theta}}}\right) \circ\left(\frac{\tilde{s}}{\sqrt{\mu_{\theta}}}\right)\right)_{\theta}^{-\frac{1}{2}} \circ \varphi^{\prime}\left(\left(\left(\frac{\tilde{x}}{\sqrt{\mu_{\theta}}}\right) \circ\left(\frac{\tilde{s}}{\sqrt{\mu_{\theta}}}\right)\right)_{\theta}^{\frac{1}{2}}\right)\right)_{\theta}\right]^{-1},
$$

and

$$
\underline{z}=\varphi\left(\left(\left(\frac{\tilde{x}}{\sqrt{\mu_{\theta}}}\right) \circ\left(\frac{\tilde{s}}{\sqrt{\mu_{\theta}}}\right)\right)_{\theta}^{\frac{1}{2}}\right)-\varphi\left(\left(\left(\frac{\tilde{x}}{\sqrt{\mu_{\theta}}}\right) \circ\left(\frac{\tilde{s}}{\sqrt{\mu_{\theta}}}\right)\right)_{\theta}\right) .
$$

If we define

$$
d_{x}=\frac{\Delta \tilde{x}}{\sqrt{\mu_{\theta}}}=\frac{P_{\theta}\left(w^{-\frac{1}{2}}\right) \Delta x}{\sqrt{\mu_{\theta}}}, \quad d_{s}=\frac{\Delta \tilde{s}}{\sqrt{\mu_{\theta}}}=\frac{P_{\theta}\left(w^{\frac{1}{2}}\right) \Delta s}{\sqrt{\mu_{\theta}}},
$$

then system (9) can be simply written in the following form:

$$
\begin{aligned}
\sqrt{\mu_{\theta}} \tilde{A} d_{x} & =0 \\
\sqrt{\mu_{\theta}} \tilde{A}^{T} \Delta y+I_{\theta, n}^{2} d_{s} & =0 \\
d_{x}+d_{s} & =p_{v_{\theta}},
\end{aligned}
$$

where

$$
p_{v_{\theta}}=\left(v_{\theta}^{-1} \circ\left(\left[\varphi^{\prime}\left(v_{\theta}^{2}\right)-\frac{1}{2}\left(v_{\theta}^{-1} \circ \varphi^{\prime}\left(v_{\theta}\right)\right)_{\theta}\right]^{-1} \circ\left(\varphi\left(v_{\theta}\right)-\varphi\left(v_{\theta}^{2}\right)\right)\right)_{\theta}\right)_{\theta} .
$$

Here, we restrict our attention to the case where $\varphi(t)=t^{2}$ based on Darvay and Takács's method for LO [5], so we have

$$
p_{v_{\theta}}=\left(\left(2 v_{\theta}^{2}-e\right)^{-1} \circ\left(v_{\theta}-\left(v_{\theta}^{2} \circ v_{\theta}\right)_{\theta}\right)\right)_{\theta} .
$$

We obtain the search directions $d_{x}$ and $d_{s}$ by solving (12) and then compute $\Delta x$ and $\Delta s$ via (11). The new iterates are obtained by taking a full-NT step as follows:

$$
x_{+}:=x+\Delta x, \quad y_{+}:=y+\Delta y, \quad s_{+}:=s+\Delta s .
$$




\subsection{The generic primal-dual algorithm for CCO}

In this section, we will show that the algorithm can solve the CCO in polynomial time. In order to we need to a quantity that measure the distance between the given triple $(x, y, s)$ and the $\mu_{\theta}$-center $\left(x\left(\mu_{\theta}\right), y\left(\mu_{\theta}\right), s\left(\mu_{\theta}\right)\right.$. We use a norm-based proximity measure as follows:

$$
\delta\left(x, s ; \mu_{\theta}\right):=\delta\left(v_{\theta}\right):=\frac{1}{2}\left\|p_{v_{\theta}}\right\|_{\theta, F}=\frac{1}{2}\left\|\left(\left(2 v_{\theta}^{2}-e\right)^{-1} \circ\left(v_{\theta}-\left(v_{\theta}^{2} \circ v_{\theta}\right)_{\theta}\right)\right)_{\theta}\right\|_{\theta, F} .
$$

From the first two equations of the system (12) it follows that

$$
d_{x} I_{\theta, n}^{2} d_{s}=\left\langle d_{x}, d_{s}\right\rangle_{\theta}=0,
$$

that is $d_{x}$ and $d_{s}$ with respect to $\theta$ are orthogonal. Let $q_{v_{\theta}}=d_{x}-d_{s}$. Then, we have

$$
\left(d_{x} \circ d_{s}\right)_{\theta}=\frac{\left(p_{v_{\theta}} \circ p_{v_{\theta}}\right)_{\theta}-\left(q_{v_{\theta}} \circ q_{v_{\theta}}\right)_{\theta}}{4} .
$$

Noting that $d_{x}$ and $d_{s}$ are orthogonal, we get $\left\|q_{v_{\theta}}\right\|_{\theta, F}=\left\|p_{v_{\theta}}\right\|_{\theta, F}=2 \delta\left(v_{\theta}\right)$. The full-NT step algorithm is given in Figure 1.

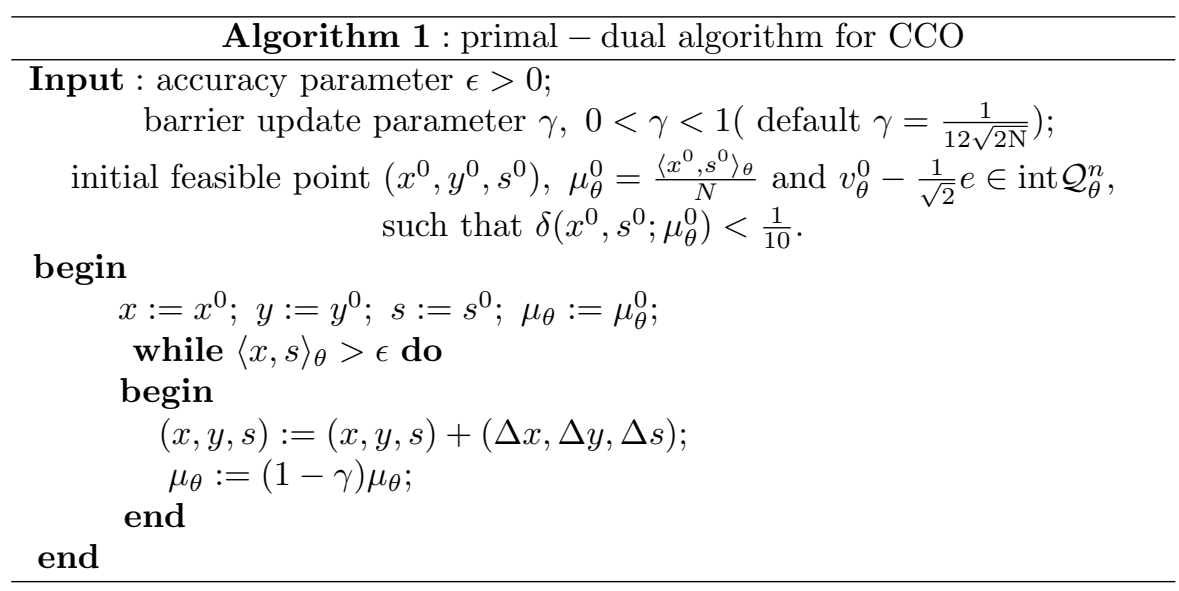

Figure 1: Primal-dual algorithm

\section{Analysis of the algorithm}

Let $x, s \in \operatorname{int} \mathcal{Q}_{\theta}^{n}, \mu_{\theta}>0$ and $w$ be the NT-scaling point of $x$ and $s$. Then, by the definition of $v_{\theta},(11)$ and (13) we get

$$
x_{+}=\sqrt{\mu_{\theta}} P_{\theta}\left(w^{\frac{1}{2}}\right)\left(v_{\theta}+d_{x}\right), \quad s_{+}=\sqrt{\mu_{\theta}} P_{\theta}\left(w^{-\frac{1}{2}}\right)\left(v_{\theta}+d_{s}\right) .
$$

Due to [2, Theorem 9] and the above relations, $x_{+}$and $s_{+}$belong to int $\mathcal{Q}_{\theta}^{n}$ if and only if $v_{\theta}+d_{x}$ and $v_{\theta}+d_{s}$ belong to int $\mathcal{Q}_{\theta}^{n}$. From now on, we denote $x-y \in \operatorname{int} \mathcal{Q}_{\theta}^{n}$ by $x \succ_{\mathcal{Q}_{\theta}^{n}} y\left(x-y \in \mathcal{Q}_{\theta}^{n}\right.$ by $x \succcurlyeq_{\mathcal{Q}_{\theta}^{n}} y$ ). Moreover, we have

$$
\begin{aligned}
v_{\theta}^{2}+\left(v_{\theta} \circ p_{v_{\theta}}\right)_{\theta}=v_{\theta}^{2}+\left(v_{\theta} \circ\left(\left(2 v_{\theta}^{2}-e\right)^{-1} \circ\left(v_{\theta}-\left(v_{\theta}^{2} \circ v_{\theta}\right)_{\theta}\right)\right)_{\theta}\right)_{\theta} \\
\quad=\left(\left(2 v_{\theta}^{2}-e\right)^{-1} \circ\left(\left(\left(2 v_{\theta}^{2}-e\right) \circ v_{\theta}^{2}\right)_{\theta}+\left(v_{\theta} \circ\left(v_{\theta}-\left(v_{\theta}^{2} \circ v_{\theta}\right)_{\theta}\right)\right)_{\theta}\right)\right)_{\theta} \\
\quad=\left(\left(2 v_{\theta}^{2}-e\right)^{-1} \circ\left(v_{\theta}^{2} \circ v_{\theta}^{2}\right)_{\theta}\right)_{\theta}-\left(\left(2 v_{\theta}^{2}-e\right)^{-1} \circ\left(2 v_{\theta}^{2}-e\right)\right)_{\theta}+e \\
\quad=\left(\left(2 v_{\theta}^{2}-e\right)^{-1} \circ\left(v_{\theta}^{2}-e\right)^{2}\right)_{\theta}+e .
\end{aligned}
$$


The following lemma states that, under some mild conditions on the proximity measure, the full-NT steps lie in $\operatorname{int} \mathcal{Q}_{\theta}^{n}$.

Lemma 1. Let $\delta_{\theta}:=\delta\left(v_{\theta}\right)<1$ and $v_{\theta} \succ_{\mathcal{Q}_{\theta}^{n}} \frac{1}{\sqrt{2}}$ e. Then the full-NT step is strictly feasible.

Proof. Let $0 \leq \alpha \leq 1$. Define $v_{x}(\alpha)=v_{\theta}+\alpha d_{x}$ and $v_{s}(\alpha)=v_{\theta}+\alpha d_{s}$. Then, using (15) and (17), we obtain

$$
\begin{aligned}
\left(v_{x}(\alpha) \circ v_{s}(\alpha)\right)_{\theta} & =\left(\left(v_{\theta}+\alpha d_{x}\right) \circ\left(v_{\theta}+\alpha d_{s}\right)\right)_{\theta} \\
& =v_{\theta}^{2}+\alpha\left(v_{\theta} \circ\left(d_{x}+d_{s}\right)\right)_{\theta}+\alpha^{2}\left(d_{x} \circ d_{s}\right)_{\theta} \\
& =(1-\alpha) v_{\theta}^{2}+\alpha\left(v_{\theta}^{2}+\left(v_{\theta} \circ p_{v_{\theta}}\right)_{\theta}\right)+\alpha^{2}\left(d_{x} \circ d_{s}\right)_{\theta} \\
& \succcurlyeq_{\mathcal{Q}_{\theta}^{n}}(1-\alpha) v_{\theta}^{2}+\alpha e+\alpha^{2}\left(d_{x} \circ d_{s}\right)_{\theta} \\
& \succcurlyeq_{\mathcal{Q}_{\theta}^{n}}(1-\alpha) v_{\theta}^{2}+\alpha\left(e-(1-\alpha) \frac{\left(p_{v_{\theta}} \circ p_{v_{\theta}}\right)_{\theta}}{4}-\alpha \frac{\left(q_{v_{\theta}} \circ q_{v_{\theta}}\right)_{\theta}}{4}\right) .
\end{aligned}
$$

Furthermore, since $0 \leq \alpha \leq 1$, we have

$$
\begin{aligned}
\left\|(1-\alpha) \frac{\left(p_{v_{\theta}} \circ p_{v_{\theta}}\right)_{\theta}}{4}+\alpha \frac{\left(q_{v_{\theta}} \circ q_{v_{\theta}}\right)_{\theta}}{4}\right\|_{\theta, F} & \leq(1-\alpha)\left\|\frac{\left(p_{v_{\theta}} \circ p_{v_{\theta}}\right)_{\theta}}{4}\right\|_{\theta, F} \\
& \leq\left(1\left\|\frac{\left(q_{v_{\theta}} \circ q_{v_{\theta}}\right)_{\theta}}{4}\right\|_{\theta, F}\right. \\
& \leq \delta_{\theta}^{2}<1 .
\end{aligned}
$$

From the last inequality, we deduce that

$$
e-(1-\alpha) \frac{\left(p_{v_{\theta}} \circ p_{v_{\theta}}\right)_{\theta}}{4}-\alpha \frac{\left(q_{v_{\theta}} \circ q_{v_{\theta}}\right)_{\theta}}{4} \succ_{\mathcal{Q}_{\theta}^{n}} 0 .
$$

Therefore, since $v_{\theta}^{2} \succ_{Q_{\theta}^{n}} 0$, we have $\left(v_{x}(\alpha) \circ v_{s}(\alpha)\right)_{\theta} \succ_{Q_{\theta}^{n}} 0$. Hence,

$$
0<\operatorname{det}_{\theta}\left(\left(v_{x}(\alpha) \circ v_{s}(\alpha)\right)_{\theta}\right) \leq \operatorname{det}_{\theta}\left(v_{x}(\alpha)\right) \operatorname{det}_{\theta}\left(v_{s}(\alpha)\right),(\text { see Lemma } 6 \text { in }[11]) .
$$

From this inequality, it follows that $\operatorname{det}_{\theta}\left(v_{x}(\alpha)\right) \neq 0$ and $\operatorname{det}_{\theta}\left(v_{s}(\alpha)\right) \neq 0$. From an argument similar to the proof of [11, Lemma 9], it follows that $v_{\theta}+d_{x} \succ_{\mathcal{Q}_{\theta}^{n}} 0$ and $v_{\theta}+d_{s} \succ \mathcal{Q}_{\theta}^{n}$. Therefore, the proof is complete.

Let us define

$$
v_{\theta}^{+}=\frac{P_{\theta}\left(w_{+}^{-\frac{1}{2}}\right) x_{+}}{\sqrt{\mu_{\theta}}}\left[=\frac{P_{\theta}\left(w_{+}^{\frac{1}{2}}\right) s_{+}}{\sqrt{\mu_{\theta}}}\right],
$$

where $w_{+}$is the NT-scaling point of $x_{+}$and $s_{+}$. Then, we have $\left(v_{\theta}^{+}\right)^{2} \sim P_{\theta}\left(\left(v_{\theta}+d_{x}\right)^{\frac{1}{2}}\right)\left(v_{\theta}+d_{s}\right)$ ([11, Lemma 11]).

Lemma 2. Let $\delta_{\theta}:=\delta\left(x, s, \mu_{\theta}\right)$. Then, we have

$$
\lambda_{\theta, \min }\left(v_{\theta}^{+}\right) \geq \sqrt{1-\delta_{\theta}^{2}}
$$


Proof. We have

$$
\begin{aligned}
\lambda_{\theta, \min }\left(v_{\theta}^{+}\right)^{2} & =\lambda_{\theta, \min }\left(P_{\theta}\left(\left(v_{\theta}+d_{x}\right)^{\frac{1}{2}}\right)\left(v_{\theta}+d_{s}\right)\right) \\
& \geq \lambda_{\theta, \min }\left(\left(\left(v_{\theta}+d_{x}\right) \circ\left(v_{\theta}+d_{s}\right)\right)_{\theta}\right) \\
& \geq \lambda_{\theta, \min }\left(e-\frac{\left(q_{v_{\theta}} \circ q_{v_{\theta}}\right)_{\theta}}{4}\right) \geq 1-\lambda_{\theta, \max }\left(\frac{\left(q_{v_{\theta}} \circ q_{v_{\theta}}\right)_{\theta}}{4}\right) \\
& \geq 1-\frac{\left\|\left(q_{v_{\theta}} \circ q_{v_{\theta}}\right)_{\theta}\right\|_{\theta, F}}{4} \geq 1-\frac{\left\|q_{v_{\theta}}\right\|_{\theta, F}^{2}}{4}=1-\delta_{\theta}^{2},
\end{aligned}
$$

where the first inequality is due to Lemma 4.58 [7], the second inequality follows from the proof of Lemma 1 with $\alpha=1$ and the fourth inequality follows from the fact that $\lambda_{\max }(x) \leq\|x\|_{F}$. The proof of the lemma is complete.

We will often use the following inequality on the circular inner product of two elements of $\mathcal{Q}_{\theta}^{n}$.

$$
\begin{aligned}
\left\|(x \circ y)_{\theta}\right\|_{\theta, F}^{2} & =\lambda_{\max , \theta}^{2}(x) \lambda_{\max , \theta}^{2}(y)+\lambda_{\min , \theta}^{2}(x) \lambda_{\min , \theta}^{2}(y) \\
& \leq \lambda_{\max , \theta}^{2}(x)\left(\lambda_{\max , \theta}^{2}(y)+\lambda_{\min , \theta}^{2}(y)\right) \\
& =\lambda_{\max , \theta}^{2}(x)\|y\|_{\theta, F}^{2}
\end{aligned}
$$

Lemma 3. Let $\delta_{\theta}:=\delta\left(x, s, \mu_{\theta}\right)<\frac{1}{\sqrt{2}}$ and $v_{\theta} \succ_{\mathcal{Q}_{\theta}^{n}} \frac{1}{\sqrt{2}} e$. Then $v_{\theta}^{+} \succ_{\mathcal{Q}_{\theta}^{n}} \frac{1}{\sqrt{2}} e$ and

$$
\delta\left(v_{\theta}^{+}\right):=\delta\left(x_{+}, s_{+} ; \mu_{\theta}\right) \leq \frac{5 \delta_{\theta}^{2}}{1-2 \delta_{\theta}^{2}} \sqrt{1-\delta_{\theta}^{2}}
$$

Proof. In view of Lemma 2, we deduce that

$$
\lambda_{\theta, \min }\left(v_{\theta}^{+}\right) \geq \sqrt{1-\delta_{\theta}^{2}}>\sqrt{1-\frac{1}{2}}=\frac{1}{\sqrt{2}} .
$$

From this inequality, it follows that $v_{\theta}^{+} \succ \mathcal{Q}_{\theta}^{n} \frac{1}{\sqrt{2}} e$. To prove the second claim, by the definition of $\delta_{\theta}$, we have

$$
\begin{aligned}
2 \delta\left(v_{\theta}^{+}\right)=\left\|p_{v_{\theta}^{+}}\right\|_{\theta, F} & =\left\|\left(\left(2\left(v_{\theta}^{+}\right)^{2}-e\right)^{-1} \circ\left(v_{\theta}^{+}-\left(v_{\theta}^{+} \circ\left(v_{\theta}^{+}\right)^{2}\right)_{\theta}\right)\right)_{\theta}\right\|_{\theta, F} \\
& =\left\|\left(\left(\left(2\left(v_{\theta}^{+}\right)^{2}-e\right)^{-1} \circ v_{\theta}^{+}\right)_{\theta} \circ\left(e-\left(v_{\theta}^{+}\right)^{2}\right)\right)_{\theta}\right\|_{\theta, F} \\
& \leq \lambda_{\max , \theta}\left(\left(\left(2\left(v_{\theta}^{+}\right)^{2}-e\right)^{-1} \circ v_{\theta}^{+}\right)_{\theta}\right)\left\|e-\left(v_{\theta}^{+}\right)^{2}\right\|_{\theta, F} .
\end{aligned}
$$

Let us consider the function $f(t)=\frac{t}{2 t^{2}-1}$ on $t>\frac{1}{\sqrt{2}}$. It is easy to see that $f^{\prime}(t)<0$, so the function $f(t)$ is decreasing. Thus, using Lemma 2, we get

$$
2 \delta\left(v_{\theta}^{+}\right) \leq \frac{\lambda_{\min , \theta}\left(v_{\theta}^{+}\right)}{2 \lambda_{\min , \theta}^{2}\left(v_{\theta}^{+}\right)-1}\left\|e-\left(v_{\theta}^{+}\right)^{2}\right\|_{\theta, F} \leq \frac{\sqrt{1-\delta_{\theta}^{2}}}{1-2 \delta_{\theta}^{2}}\left\|e-\left(v_{\theta}^{+}\right)^{2}\right\|_{\theta, F} .
$$


On the other hand, using [15, Lemma 30], we get

$$
\begin{aligned}
\left\|\left(v_{\theta}^{+}\right)^{2}-e\right\|_{\theta, F} & =\left\|P_{\theta}\left(\left(v_{\theta}+d_{x}\right)^{\frac{1}{2}}\right)\left(v_{\theta}+d_{s}\right)-e\right\|_{\theta, F} \\
& \leq\left\|\left(\left(v_{\theta}+d_{x}\right) \circ\left(v_{\theta}+d_{s}\right)\right)_{\theta}-e\right\|_{\theta, F} \\
& =\left\|v_{\theta}^{2}+\left(v_{\theta} \circ p_{v_{\theta}}\right)_{\theta}+\left(d_{x} \circ d_{s}\right)_{\theta}-e\right\|_{\theta, F} \\
& =\left\|\left(\left(2 v_{\theta}^{2}-e\right)^{-1} \circ\left(v_{\theta}^{2}-e\right)^{2}\right)_{\theta}+\frac{\left(p_{v_{\theta}} \circ p_{v_{\theta}}\right)_{\theta}-\left(q_{v_{\theta}} \circ q_{v_{\theta}}\right)_{\theta}}{4}\right\|_{\theta, F} \\
& =\left\|\left(\left(v_{\theta}^{-2} \circ\left(9 v_{\theta}^{2}-4 e\right)\right)_{\theta} \circ\left(\frac{\left(p_{v_{\theta}} \circ p_{v_{\theta}}\right)_{\theta}}{4}\right)\right)_{\theta}-\frac{\left(q_{v_{\theta}} \circ q_{v_{\theta}}\right)_{\theta}}{4}\right\|_{\theta, F} \\
\leq & \lambda_{\max , \theta}\left(\left(v_{\theta}^{-2} \circ\left(9 v_{\theta}^{2}-4 e\right)\right)_{\theta}\right)\left\|\frac{\left(p_{v_{\theta}} \circ p_{v_{\theta}}\right)_{\theta}}{4}\right\|_{\theta, F}+\left\|\frac{\left(q_{v_{\theta}} \circ q_{v_{\theta}}\right)_{\theta}}{4}\right\|_{\theta, F} \\
& \leq 9 \frac{\left\|p_{v_{\theta}}\right\|_{\theta, F}^{2}}{4}+\frac{\left\|q_{v_{\theta}}\right\|_{\theta, F}^{2}}{4}=10 \delta_{\theta}^{2}
\end{aligned}
$$

where the second equality is due to the proof of Lemma 1 with $\alpha=1$, the third equality follows from (15) and (17). Substituting this bound into (19) yields the inequality in lemma, and the proof is complete.

The next lemma gives an upper bound of the duality gap after taking a full-NT step.

Lemma 4. Let $\delta_{\theta}:=\delta\left(x, s ; \mu_{\theta}\right)$. Then, we have

$$
\left\langle x_{+}, s_{+}\right\rangle_{\theta} \leq \mu_{\theta}\left(N+4 \delta_{\theta}^{2}\right) .
$$

Proof. By the circular inner product and (16) it follows that

$$
\begin{aligned}
\left\langle x_{+}, s_{+}\right\rangle_{\theta}=x_{+}^{T} I_{\theta, n}^{2} s_{+} & =\mu_{\theta}\left(P_{\theta}\left(w^{\frac{1}{2}}\right)\left(v_{\theta}+d_{x}\right)\right)^{T} I_{\theta, n}^{2} P_{\theta}\left(w^{-\frac{1}{2}}\right)\left(v_{\theta}+d_{s}\right) \\
= & \mu_{\theta}\left(v_{\theta}+d_{x}\right)^{T} P_{\theta}\left(w^{\frac{1}{2}}\right)^{T} I_{\theta, n}^{2} P_{\theta}\left(w^{-\frac{1}{2}}\right)\left(v_{\theta}+d_{s}\right) \\
= & \mu_{\theta}\left(v_{\theta}+d_{x}\right)^{T} I_{\theta, n}^{2} P_{\theta}\left(w^{\frac{1}{2}}\right) P_{\theta}\left(w^{-\frac{1}{2}}\right)\left(v_{\theta}+d_{s}\right) \\
& =\mu_{\theta}\left\langle v_{\theta}+d_{x}, v_{\theta}+d_{s}\right\rangle_{\theta} \\
& =\mu_{\theta}\left(\left\langle v_{\theta}, v_{\theta}\right\rangle_{\theta}+\left\langle v_{\theta}, d_{x}+d_{s}\right\rangle_{\theta}+\left\langle d_{x}, d_{s}\right\rangle_{\theta}\right) \\
& =\mu_{\theta}\left(\left\langle v_{\theta}, v_{\theta}\right\rangle_{\theta}+\left\langle v_{\theta}, p_{v_{\theta}}\right\rangle_{\theta}\right)=\frac{\mu_{\theta}}{2} \operatorname{tr}\left(v_{\theta}^{2}+\left(v_{\theta} \circ p_{v_{\theta}}\right)_{\theta}\right) \\
& =\frac{\mu_{\theta}}{2} \operatorname{tr}\left(\left(\left(2 v_{\theta}^{2}-e\right)^{-1} \circ\left(v_{\theta}^{2}-e\right)^{2}\right)_{\theta}+e\right) \\
& =\frac{\mu_{\theta}}{2} \operatorname{tr}\left(\left(\left(v_{\theta}^{-2} \circ\left(2 v_{\theta}^{2}-e\right)\right)_{\theta} \circ\left(p_{v_{\theta}} \circ p_{v_{\theta}}\right)_{\theta}\right)_{\theta}+e\right) \\
& \leq \frac{\mu_{\theta}}{2}\left(\lambda_{\max , \theta}\left(\left(v_{\theta}^{-2} \circ\left(2 v_{\theta}^{2}-e\right)\right)_{\theta}\right) \operatorname{tr}\left(\left(p_{v_{\theta}} \circ p_{v_{\theta}}\right)_{\theta}\right)+2 N\right) \\
& \leq \frac{\mu_{\theta}}{2}\left(\frac{8\left\|p_{v_{\theta}}\right\|_{\theta, F}^{2}}{4}+2 N\right)=\mu_{\theta}\left(N+4 \delta_{\theta}^{2}\right),
\end{aligned}
$$

where the third equality follows from $\left(I_{\theta, n}^{2} P_{\theta}\left(w^{\frac{1}{2}}\right)\right)^{T}=I_{\theta, n}^{2} P_{\theta}\left(w^{\frac{1}{2}}\right)$ (see [11]), the sixth equality is due to the third equation of (12) and the fact that $d_{x}$ and $d_{s}$ are orthogonal. The proof of the lemma is complete.

Lemma 5. Let $\delta_{\theta}:=\delta\left(x, s, \mu_{\theta}\right)<\frac{1}{\sqrt{2}}, v_{\theta} \succ_{\mathcal{Q}_{\theta}^{n}} \frac{1}{\sqrt{2}} e$ and $\mu_{\theta}^{+}:=(1-\gamma) \mu_{\theta}$ with $0<\gamma<1$. Furthermore, let $v_{\theta}^{\sharp}=\frac{v_{\theta}^{+}}{\sqrt{1-\gamma}}$. Then, $v_{\theta}^{\sharp} \succ \mathcal{Q}_{\theta}^{n} \frac{1}{\sqrt{2}}$ e and

$$
\delta\left(x_{+}, s_{+} ; \mu_{\theta}^{+}\right) \leq \frac{\sqrt{1-\delta_{\theta}^{2}}}{2 \sqrt{1-\gamma}\left(1-2 \delta_{\theta}^{2}+\gamma\right)}\left(\gamma \sqrt{2 N}+10 \delta_{\theta}^{2}\right) .
$$


Proof. Under the assumptions given in lemma, we conclude from Lemma 3 that $v_{\theta}^{+} \succ \mathcal{Q}_{\theta}^{n} \frac{1}{\sqrt{2}} e$. This, together with $\frac{1}{\sqrt{1-\gamma}}>1$, implies that $v_{\theta}^{\sharp} \succ \mathcal{Q}_{\theta}^{n} \frac{1}{\sqrt{2}} e$, and we have proved the first statement. To prove the second claim, by the definition of $\delta_{\theta}$, we have

$$
\begin{aligned}
& \delta\left(x_{+}, s_{+} ; \mu_{\theta}^{+}\right)=\frac{1}{2}\left\|\left(\left(2\left(v_{\theta}^{\sharp}\right)^{2}-e\right)^{-1} \circ\left(v_{\theta}^{\sharp}-\left(v_{\theta}^{\sharp} \circ\left(v_{\theta}^{\sharp}\right)^{2}\right)_{\theta}\right)\right)_{\theta}\right\|_{\theta, F} \\
& \quad=\frac{1}{2 \sqrt{1-\gamma}}\left\|\left(\left(2\left(v_{\theta}^{+}\right)^{2}-e(1-\gamma)\right)^{-1} \circ\left(v_{\theta}^{+} \circ\left((1-\gamma) e-\left(v_{\theta}^{+}\right)^{2}\right)\right)_{\theta}\right)_{\theta}\right\|_{\theta, F} \\
& \quad \leq \frac{1}{2 \sqrt{1-\gamma}} \lambda_{\max , \theta}\left(\left(\left(2\left(v_{\theta}^{+}\right)^{2}-e(1-\gamma)\right)^{-1} \circ v_{\theta}^{+}\right)_{\theta}\right)\left\|(1-\gamma) e-\left(v_{\theta}^{+}\right)^{2}\right\|_{\theta, F} \\
& \leq \frac{\sqrt{1-\delta_{\theta}^{2}}}{2 \sqrt{1-\gamma}\left(1-2 \delta_{\theta}^{2}+\gamma\right)}\left(\gamma \sqrt{2 N}+\left\|e-\left(v_{\theta}^{+}\right)^{2}\right\|_{\theta, F}\right) \\
& \quad \leq \frac{\sqrt{1-\delta_{\theta}^{2}}}{2 \sqrt{1-\gamma}\left(1-2 \delta_{\theta}^{2}+\gamma\right)}\left(\gamma \sqrt{2 N}+10 \delta_{\theta}^{2}\right),
\end{aligned}
$$

which concludes the proof of lemma.

Corollary 1. Let $\delta_{\theta}:=\delta\left(x, s, \mu_{\theta}\right)<\frac{1}{10}$ and $\gamma=\frac{1}{12 \sqrt{2 N}}$. Then $\delta\left(x_{+}, s_{+} ; \mu_{\theta}^{+}\right)<\frac{1}{10}$.

The following lemma gives an upper bound for the number of iterations produced by Algorithm.

Lemma 6. Suppose that $x^{0}$ and $s^{0}$ are strictly feasible, $\mu_{\theta}^{0}=\frac{\left\langle x^{0}, s^{0}\right\rangle_{\theta}}{N}$ and $\delta\left(x^{0}, s^{0} ; \mu_{\theta}^{0}\right)<\frac{1}{10}$. Let $x^{k}$ and $s^{k}$ be the iterates obtained after $k$ iterations. Then, $\left\langle x^{k}, s^{k}\right\rangle_{\theta} \leq \varepsilon$ for any

$$
k \geq\left\lceil\frac{1}{\gamma} \log \frac{\mu_{\theta}^{0}\left(N+\frac{1}{25}\right)}{\varepsilon}\right\rceil .
$$

Proof. From Lemma 4 and the assumption $\delta\left(x, s ; \mu_{\theta}\right)<\frac{1}{10}$, we conclude that

$$
\left\langle x^{k}, s^{k}\right\rangle_{\theta} \leq \mu_{\theta}^{k}\left(N+\frac{1}{25}\right)=(1-\gamma)^{k} \mu_{\theta}^{0}\left(N+\frac{1}{25}\right) .
$$

Then, the inequality $\left\langle x^{k}, s^{k}\right\rangle_{\theta} \leq \varepsilon$ holds if

$$
(1-\gamma)^{k} \mu_{\theta}^{0}\left(N+\frac{1}{25}\right) \leq \varepsilon
$$

By taking the natural logarithm of both sides of (19), we get

$$
k \log (1-\gamma)+\log \mu_{\theta}^{0}\left(N+\frac{1}{25}\right) \leq \log \varepsilon .
$$

Using the inequality $-\log (1-\gamma)>\gamma$, we conclude that the above inequality is satisfied if

$$
-k \gamma+\log \mu_{\theta}^{0}\left(N+\frac{1}{25}\right) \leq \log \varepsilon
$$

which implies the desired result.

We are now in a position to state the main result of this paper.

Theorem 1. Let $\gamma=\frac{1}{12 \sqrt{2 N}}$. Then the algorithm requires at most

$$
\mathcal{O}\left(\sqrt{N} \log \frac{\mu_{\theta}^{0}\left(N+\frac{1}{25}\right)}{\varepsilon}\right)
$$

iterations. The output gives an $\varepsilon$-approximate solution $(x, s)$ satisfying $\langle x, s\rangle_{\theta} \leq \varepsilon$.

Proof. The proof is trivial by Lemma 6 . 


\section{Conclusion}

In this paper, we proposed an IPM for circular cone optimization based on Darvay and Takács's techique for LO [5] and using the Nesterov-Todd (NT) symmetrization scheme. We used Newton's method to get the search directions and showed that the obtained complexity coincides with the now best-known iteration bound for small-update methods. As a further research one can consider the possible extension of the proposed method to the elliptic cone optimization.

\section{References}

[1] Achache, M. (2006). A new primal-dual path-following method for convex quadratic programming. Computational and Applied Mathematics, 25(1), 97-110. doi: 10.1590/s0101-82052006000100005

[2] Alizadeh, F. and Goldfarb, D. (2003). Second-order cone programming. Mathematical Programming, 95(1), 3-51. doi: 10.1007/s10107-002-0339-5

[3] Alzalg, B. (2017). The Jordan algebraic structure of the circular cone. Operators and Matrices, 11(1), 1-21. doi: 10.7153/oam-11-01

[4] Darvay, Z. (2003). New interior point algorithms in linear programming. Advanced Modeling and Optimization, 5(1), 51-92. https://camo.ici.ro/journal/vol5/v5a4.pdf

[5] Darvay, Z. and Takács, P. R. (2018). New method for determining search directions for interior-point algorithms in linear optimization. Optimization Letters, 12(5), 1099-1116. doi: 10.1007/s11590-017-1171-4

[6] De Klerk, E. (2002). Aspects of Semidefinite Programming. Applied Optimization. Springer, Boston. doi: $10.1007 / \mathrm{b} 105286$

[7] Gu, G. (2009). Full-step interior-point methods for symmetric optimization. PhD Thesis, TU Delft.

[8] Illés, T. and Terlaky, T. (2002). Pivot versus interior point methods: Pros and cons. European Journal of Operational Research, 140(2), 170-190. doi: 10.1016/s0377-2217(02)00061-9

[9] Karmarkar, N. K. (1984). A new polynomial-time algorithm for linear programming. Combinatorica, 4(4), 373-395. doi: 10.1007/bf02579150

[10] Kheirfam, B. (2014). A new complexity analysis for full-Newton step infeasible interior-point algorithm for horizontal linear complementarity problems. Journal of Optimization Theory and Applications, 161(3), 853-869. doi: 10.1007/s10957-013-0457-7

[11] Kheirfam, B. (2016). A full Nesterov-Todd step interior-point method for circular cone optimization. Communications in Combinatorics and Optimization, 1(2), 83-102. doi: 10.22049 /cco.2016.13554

[12] Ko, C. H. and Chen, J. S. (2013). Optimal grasping manipulation for multifingered robots using semismooth Newton method. Mathematical Problems in Engineering, 2013(ID 681710), 1-9. doi: $10.1155 / 2013 / 681710$

[13] Monteiro, R. D. C. (1997). Primal-dual path-following algorithms for semidefinite programming. SIAM Journal on Optimization, 7(3), 663-678. doi: 10.1137/s1052623495293056

[14] Roos, C., Terlaky, T. and Vial, J-Ph. (1997). Theory and Algorithms for Linear Optimization. An Interior-Point Approach. John Wiley \& Sons, Chichester.

[15] Schmieta, S. H. and Alizadeh, F. (2003). Extension of primal-dual interior point methods to symmetric cones. Mathematical Programming, 96(3), 409-438. doi: 10.1007/s10107-003-0380-z

[16] Wang, G. Q. and Lesaja, G. (2013). Full Nesterov-Todd step feasible interior-point method for the Cartesian $P_{*}(\kappa)-$ SCLCP. Optimization Methods and Software, 28(3), 600-618. doi: 10.1080/10556788.2013.781600

[17] Wang, G. Q. and Bai, Y. Q. (2009). A primal-dual interior-point algorithm for second-order cone optimization with full Nesterov-Todd step. Applied Mathematics and Computation, 215(3), 10471061. doi: 10.1016/j.amc.2009.06.034

[18] Wang, G. Q. and Bai, Y. Q. (2009). A new primal-dual path-following interior-point algorithm for semidefinite optimization. Journal of Mathematical Analysis and Applications, 353(1), 339-349. doi: $10.1016 /$ j.jmaa.2008.12.016

[19] Wang, G. Q. and Bai, Y. Q. (2012). A new full NesterovTodd step primal-dual path-following interior-point algorithm for symmetric optimization. Journal of Optimization Theory and Applications, 154(3), 966-985. doi: 10.1007/s10957-012-0013-x 
[20] Wang, G. Q., Yu, C. J. and Teo, K. L. (2014). A full-Newton step feasible interior-point algorithm for $P_{*}(\kappa)$-linear complementarity problem. Journal of Global Optimization, 59(1), 81-99. doi: 10.1007/s10898-013-0090-x

[21] Wang, G. Q., Fan, X. J., Zhu, D. T. and Wang, D. Z. (2015). New complexity analysis of a full-Newton step infeasible interior-point algorithm for $P_{*}(\kappa)$-LCP. Optimization Letters, $9(6)$, 1105-1119. doi: 10.1007/s11590-014-0800-4

[22] Zhang, L., Sun, L. and Xu, Y. (2013). Simplified analysis for full-Newton step infeasible interior-point algorithm for semidefinite programming. Optimization, 62(2), 169-191. doi: 10.1080/02331934.2011.571257 